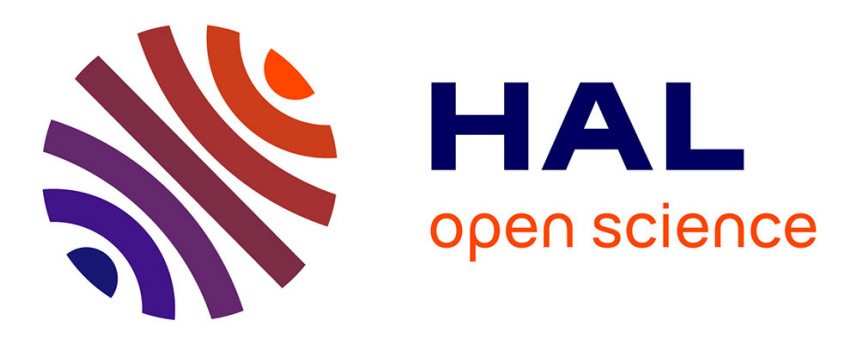

\title{
Investigations on the afterglow of a thin cathode discharge in argon at atmospheric pressure
} Sebastian Mohr, Beilei Du, Dirk Luggenhölscher, Uwe Czarnetzki

\section{To cite this version:}

Sebastian Mohr, Beilei Du, Dirk Luggenhölscher, Uwe Czarnetzki. Investigations on the afterglow of a thin cathode discharge in argon at atmospheric pressure. Journal of Physics D: Applied Physics, 2010, 43 (29), pp.295201. 10.1088/0022-3727/43/29/295201 . hal-00569656

\section{HAL Id: hal-00569656 https://hal.science/hal-00569656}

Submitted on 25 Feb 2011

HAL is a multi-disciplinary open access archive for the deposit and dissemination of scientific research documents, whether they are published or not. The documents may come from teaching and research institutions in France or abroad, or from public or private research centers.
L'archive ouverte pluridisciplinaire HAL, est destinée au dépôt et à la diffusion de documents scientifiques de niveau recherche, publiés ou non, émanant des établissements d'enseignement et de recherche français ou étrangers, des laboratoires publics ou privés. 


\title{
Investigations on the afterglow of a thin cathode discharge in argon at atmospheric pressure
}

\author{
Sebastian Mohr, Beilei Du, Dirk Luggenhölscher and Uwe Czarnetzki \\ Institute for Plasma and Atomic Physics, Ruhr-University Bochum, 44780 Bochum, Germany \\ E-mail: sebastian.mohr@ep5.rub.de
}

\begin{abstract}
A thin cathode discharge consists of two electrodes separated by a dielectric layer with a thickness of ca. $100 \mu \mathrm{m}$. The shape of the anode can be chosen arbitrarily, while the thickness of the cathode is also about $100 \mu \mathrm{m}$. Through this "sandwich", a hole with a diameter of $200 \mu \mathrm{m}$ is drilled. When such a device is operated at pressures of several $100 \mathrm{hPa}$, it shows a self-pulsing behaviour in which high electron densities of several $10^{16} \mathrm{~cm}^{-3}$ are reached. Electrical measurements showed, that this can be explained by the repeated ignition of a shortliving spark discharge. Due to the high pressure and the related high collision frequencies, the afterglow of this discharge was expected to last several $10 \mathrm{~ns}$. Instead, lifetimes of several 100 ns were observed. In order to identify the mechanisms responsible for this long living afterglow, a kinetic model of the afterglow was developed. As a result, Penning-ionization, superelastic collisions with both atoms in excited states and excimers were found to play a crucial role in the production and heating of electrons.
\end{abstract}

PACS numbers: 52.25.Dg, 52.70.Ds, 52.70.Kz, 52.75.Xx, 52.80.Mg,

Submitted to: 


\section{Introduction}

In the course of the last decade, the interest in microdischarges has constantly risen. Besides quite obvious reasons, like the reduced material cost and easy transportability, this interest stems from the possibility to operate these discharges at high pressures up to atmospheric pressure and above at relatively low supply voltages of several $100 \mathrm{~V}$ as a result of the Paschen-law. Many variations of microdischarges have been proposed and investigated including dielectric barrier discharges [1-3], microjets [4-6], the plasma needle [7,8] and the microhollow cathode discharge (MHCD) [9-18] which is the subject of this study. The applications of microdischarges exploit characteristics like the high electron density $[9,10]$ which is used to process environmentally hazardous materials [19] or to produce ions for analytical purposes [11]. Furthermore, three body processes are much more likely at high pressures which results for example in the production of ozone [20] or excimers which emit UV and VUV radiation [15].

The MHCD is a miniaturized version of the well known hollow cathode. It consists of two metal electrodes, which are a few $100 \mu \mathrm{m}$ thick, separated by a thin dielectric layer of about the same thickness. The discharge burns in a hole, whose diameter is of the same order as the thickness of the layers, drilled through this setup. The MHCD was first investigated by Schoenbach [12-16] who quickly discovered that it can be operated in different modes depending on pressure and the applied supply voltage. At relatively low pressures $(<300 \mathrm{hPa})$, there are two different continuous modes, one in which the discharge is confined inside the hole and one in which it expands onto the cathode surface, and a self-pulsing mode which was thoroughly investigated by Rousseau [17, 18]. This selfpulsing mode can be explained by a periodic transition between the two continuous modes in which the discharge on the cathode surface is repeatedly ignited, but cannot be sustained by the supplying current and thus collapses. This self-pulsing is characterized by a periodic rise and fall of the voltage and periodically occurring current peaks of some mA which last some $\mu$ s.

At pressures above $300 \mathrm{hPa}$, another kind of self-pulsing can be observed. Compared to the selfpulsing at low pressures, the changes in voltage and current are larger and occur on much shorter timescales; the voltage falls several $100 \mathrm{~V}$ down to zero in just a few ns, while the current can reach several $10 \mathrm{~A}$ in this period and then decays in a few ns. The I-V characteristic suggests, that this selfpulsing is caused by a repeated ignition of a spark discharge which instantly collapses. The feature, which makes this possible, is the thinness of the cathode (in our case $100 \mu \mathrm{m}$ ) which enables the cathode to heat very quickly and emit electrons by thermionic emission. Thus, we chose to call this mode thin cathode discharge (TCD) instead of microhollow cathode discharge.

In this TCD-mode, temporally resolved emission spectroscopic measurements have been performed. Besides simply measuring the emission intensity, we also measured the electron density via the Stark broadening of the H-Beta line [21]. These measurements showed that the afterglow of the arc 
discharge ignited during the self-pulsing has a surprisingly long lifetime of several $100 \mathrm{~ns}$ in which a high electron density of several $10^{16} \mathrm{~cm}^{-3}$ can be reached.

To explain the long living afterglow, we developed a kinetic model. It is based on the models of Rockwood [22], Bretagne [23, 24] and Elliot and Greene [25] and features the excited argon states, atomic and molecular ions and argon excimers. The electron energy distribution function (EEDF), which was assumed to be non-Maxwellian, has been explicitly calculated by solving the Boltzmannequation. Through this simulation, an understanding of the dominant processes in the afterglow has been obtained.

The first part of this paper describes the kinetic model we used for the simulation. This is followed by the presentation of the experimental setup and results. Finally, the simulation results are presented and discussed.

\section{The kinetic model of the afterglow}

\subsection{General concept of the model}

Table 1. Processes involving electrons.

\begin{tabular}{cc}
\hline Process & References \\
\hline$e+A r \leftrightarrow e+A r\left(1 s_{3}, 1 s_{5}\right)$ & {$[23]$} \\
$e+A r \leftrightarrow e+A r\left(1 s_{2}, 1 s_{4}\right)$ & {$[23]$} \\
$e+A r \leftrightarrow e+A r(2 p)$ & {$[26]$} \\
$e+A r(1 s) \leftrightarrow e+A r(2 p)$ & {$[24],[27]$} \\
$e+A r \rightarrow e+e+A r^{+}$ & {$[24]$} \\
$e+A r(1 s) \rightarrow e+e+A r^{+}$ & {$[24]$} \\
$e+A r(2 p) \rightarrow e+e+A r^{+}$ & {$[24]$} \\
$e+A r_{2}^{*} \rightarrow e+e+A r_{2}^{+}$ & {$[24]$} \\
$e+A r_{2}^{*} \rightarrow e+2 A r$ & {$[24]$} \\
$e+A r_{2}^{+} \rightarrow A r+A r(1 s, 2 p)$ & {$[24]$} \\
$e+A r_{3}^{+} \rightarrow 2 A r+A r(1 s, 2 p)$ & see text \\
$e+A r \rightarrow e+A r$ & {$[24],[25]$} \\
$e+e \rightarrow e+e$ & {$[25]$} \\
\hline
\end{tabular}

The model used in this study is based on the models of Bretagne [23, 24], Rockwood [22] and Elliot and Greene [25]. It includes electrons, $A r^{+}, A r_{2}^{+}, A r_{3}^{+}, A r_{2}^{*}$ (excimers) and excited argon levels which were treated individually. In case of the 1 s-levels, the fluorescent $1 s_{4}$ and $1 s_{2}$ levels can also be considered to be metastable due to the high ground state density and the related radiation trapping. To describe the electron kinetics, a discrete electron energy distribution function (EEDF) with an energy interval width of $0.2 \mathrm{eV}$ and a cut-off at $20 \mathrm{eV}$ was used. Smaller intervals or cut-offs at higher energies yield no significantly differing results, but lead to much longer calculation times. Since the 
ground state density $n_{g}$ is much higher than the densities of the other species, it was considered to be constant. Furthermore, this model describes the afterglow, so there is no electric field which needs to be considered.

Table 2. Atomic and molecular processes.

\begin{tabular}{lll}
\hline \multicolumn{1}{c}{ Process } & \multicolumn{1}{c}{ Rate coefficient $^{\mathrm{a}}$} & References \\
\hline $2 A r+A r^{+} \rightarrow A r+A r_{2}^{+}$ & $k_{1}=2.5 \times 10^{-31}\left(300 / T_{g}\right)$ & {$[28]$} \\
$2 A r+A r_{2}^{+} \rightarrow A r+A r_{3}^{+}$ & $k_{2}=7 \times 10^{-32}\left(300 / T_{g}\right)^{\mathrm{b}}$ & {$[29]$} \\
$A r+A r_{3}^{+} \rightarrow 2 A r+A r_{2}^{+}$ & $k_{3}=9 \times 10^{-12}$ & {$[29]$} \\
$2 A r+A r(1 s) \rightarrow A r+A r_{2}^{*}$ & $k_{4}=10^{-32}$ & {$[29]$} \\
$A r_{2}^{*} \rightarrow 2 A r+h v$ & $k_{5}=10^{6}$ & {$[28]$} \\
$A r(1 s)+A r(1 s) \rightarrow A r+A r^{+}+e$ & $k_{P e n n}=5 \times 10^{-10}\left(T_{g} / 300 \mathrm{~K}\right)^{0.5}$ & {$[28]$} \\
$A r(1 s)+A r(2 p) \rightarrow A r+A r^{+}+e$ & $k_{P e n n}=5 \times 10^{-10}\left(T_{g} / 300 \mathrm{~K}\right)^{0.5}$ & {$[28]$} \\
$A r(2 p)+A r(2 p) \rightarrow A r+A r^{+}+e$ & $k_{P e n n}=5 \times 10^{-10}\left(T_{g} / 300 \mathrm{~K}\right)^{0.5}$ & {$[28]$} \\
\hline${ }^{a} T h e$ unit is $\mathrm{cm}^{3} \mathrm{~s}^{-1}$ for two-body, $\mathrm{cm}^{6} \mathrm{~s}^{-1}$ for three-body processes and $\mathrm{s}^{-1}$ for radiative processes. \\
${ }^{\mathrm{b} T e m p e r a t u r e ~ d e p e n d e n c y ~ w a s ~ a s s u m e d ~ t o ~ b e ~ t h e ~ s a m e ~ a s ~ f o r ~ t h e ~ f o r m a t i o n ~ o f ~} \mathrm{Ar}_{2}^{+}$.
\end{tabular}

Tables 1 and 2 give an overview of some of the processes implemented in the simulation. The radiative transitions from the $2 p$ - to the 1 s-states along with the Einstein coefficients can be found in [30], quenching and population transfer processes with their rate coefficients in [28]. We divided these processes into two categories. One is composed of processes which are suspected to be responsible for the prolonging of the afterglow. These are Penning-ionization, superelastic collisions with atoms in excited states and the formation of excimers which can also be ionized or perform superelastic collisions with electrons. The other category consists of processes which are certainly not responsible for the prolonging of the afterglow, as they either cool the electrons, lead to an electron loss or do not affect the electron kinetics. We call these processes "basic" processes. They include electron impact ionization, excitation, recombination, radiation, quenching, the formation of molecular ions, population transfer and elastic collisions of the electrons with the background gas. Furthermore, Coulomb-collisions between the electrons have to be considered due to the high electron density. Usually, diffusion can be easily neglected at these high pressures, but due to the small dimensions, an estimation of the timescales on which diffusion occurs should be done. The diffusion coefficient for excited argon atoms taken from [31], $D^{*}=2.42 \times 10^{18} / \frac{n_{g}}{\left[\mathrm{~cm}^{-3}\right]} \mathrm{cm}^{2} \mathrm{~s}^{-1}$, yields diffusion times of $\sim 50$ $\mu \mathrm{s}$, assuming a cylindrical geometry and a diffusion length of $100 \mu \mathrm{m}$. Ions and electrons are subject to ambipolar diffusion; the diffusion coefficient $D_{a m b}$ can be estimated by 


$$
D_{a m b}=D_{i}\left(1+\frac{T_{e}}{T_{i}}\right)
$$

where $D_{i}$ is the diffusion coefficient for the free diffusion of argon ions and $T_{e}$ and $T_{i}$ are the electron and ion temperature. $D_{i}$ can be calculated via the Einstein-relation from the ion mobility given in [31]. Assuming, the electron temperature is of the order of $\mathrm{eV}$ and the ion temperature of the order of $0.1 \mathrm{eV}$, this estimation yields diffusion times of several $\mu \mathrm{s}$. Since the simulation was run over a period of $1 \mu \mathrm{s}$, diffusion has been neglected.

\subsection{Treatment of the Boltzmann-equation}

The Boltzmann-equation for the electrons can be separated in terms describing inelastic and elastic processes:

$$
\frac{d f(E, t)}{d t}=\left.\frac{d f}{d t}\right|_{\text {inelastic }}+\left.\frac{d f}{d t}\right|_{\text {elastic }}
$$

The inelastic term can further be separated into terms describing excitation processes, super-elastic collisions, electron impact ionization, Penning-ionization and recombination:

$$
\left.\frac{d f}{d t}\right|_{\text {inelastic }}=K_{e x c}+K_{\text {sel }}+K_{\text {ion }}+K_{P e n n}+K_{r e c}
$$

The term describing the excitation processes is

$$
K_{e x c}(E, t)=\sum_{\substack{l, k \\ l<k}} n_{l}(t) \mathrm{v}\left(E+\Delta E_{l k}\right) \sigma_{l k}\left(E+\Delta E_{l k}\right) f\left(E+\Delta E_{l k}\right)-\sum_{\substack{l, k \\ l<k}} n_{l}(t) \mathrm{v}(E) \sigma_{l k}(E) f(E),
$$

where $n_{l}$ is the density of state $1, \mathrm{v}(\mathrm{E})$ the velocity of an electron with energy $\mathrm{E}, \sigma_{l k}(E)$ the cross section for the electron impact excitation from level 1 to level $\mathrm{k}$ and $\Delta E_{l k}$ the energy difference between state 1 and $\mathrm{k}$. Thus, the first term describes the gain of electrons with energy $\mathrm{E}$ caused by excitation processes of electrons with energy $E+\Delta E_{l k}$ and the second term describes the loss of electrons with energy E. 
The term for the super-elastic collisions takes a form similar to $K_{\text {exc }}$ :

$$
K_{\text {sup }}(E, t)=\sum_{\substack{l, k \\ l<k}} n_{k}(t) \mathrm{v}\left(E-\Delta E_{k l}\right) \sigma_{k l}\left(E-\Delta E_{k l}\right) f\left(E-\Delta E_{k l}\right)-\sum_{\substack{l, k \\ l<k}} n_{k}(t) \mathrm{v}(E) \sigma_{k l}(E) f(E)
$$

The cross sections for the super-elastic collisions were calculated using the principle of microreversibility:

$$
\sigma_{k l}=\frac{g_{l}}{g_{k}} \frac{E+\Delta E_{k l}}{E} \sigma_{l k}\left(E+\Delta E_{k l}\right)
$$

where $g_{l}$ and $g_{k}$ are the statistical weights of the involved levels. For super-elastic collisions with excimers, for which no inverse process exists, the cross section given by Bretagne [24] was used.

In order to describe the ionization processes, differential cross sections $\sigma\left(E, E^{\prime}\right)$, which are dependent on the energy of the ionizing electrons $\mathrm{E}$ and the energy of the secondary electrons $E^{\prime}$, were needed. To calculate those cross sections, the formula proposed by Bretagne [24] was used. The total ionization cross section can be calculated by

$$
\sigma_{i o n, t o t}(E)=\int \sigma\left(E, E^{\prime}\right) d E^{\prime}
$$

Formulating $K_{i o n}(E)$ one has to consider the gain of electrons with energy E either by the transfer of electrons from higher energies or by secondary electrons and the loss of electrons with energy E which leads to:

$$
\begin{aligned}
K_{\text {ion }}(E, t) & =\sum_{p} n_{p}(t) \int \mathrm{v}\left(E^{\prime}\right) \sigma_{p}\left(E^{\prime}, E^{\prime}-E_{i o n}-E\right) f\left(E^{\prime}\right) d E^{\prime}+\sum_{p} n_{p}(t) \int \mathrm{v}\left(E^{\prime}\right) \sigma_{p}\left(E^{\prime}, E\right) f\left(E^{\prime}\right) d E^{\prime} \\
& -\sum_{p} n_{p}(t) \mathrm{v}(E) f(E) \int \sigma_{p}\left(E, E^{\prime}\right) d E^{\prime}
\end{aligned}
$$

The summation takes place over all species $\mathrm{p}$ which can be ionized.

The Penning-ionization is characterised by the rate coefficient $k_{P e n n}=5 \times 10^{-10}\left(T_{g} / 300 \mathrm{~K}\right)^{0.5} \mathrm{~cm}^{3} \mathrm{~s}^{-1}$ [28] which yields no information about the energy of the secondary electrons. However, Elson [29] gives the energy intervals in which $80 \%$ of the secondary electrons are created. Assuming that all secondary electrons are created in these energy intervals and are equally distributed within them, the term describing Penning-ionization then becomes: 


$$
K_{P e n n}(E, t)=\sum_{l, k} k_{P e n n} \frac{n_{l}(t) n_{k}(t)}{N w},
$$

where $n_{l, k}$ is the density of the excited state 1 or $\mathrm{k}, \mathrm{N}$ is the number of energy intervals of the discrete EEDF, which lie within the boundaries of the energy interval in which secondary electrons are created, and $\mathrm{w}$ is the width of these energy intervals.

The recombination term is given by:

$$
K_{r e c}(E, t)=-\mathrm{v}(E) f(E, t)\left(\sigma_{r e c, A r_{2}^{+}}(E) n_{A r_{2}^{+}}+\sigma_{r e c, A r_{3}^{+}}(E) n_{A r_{3}^{+}}\right) .
$$

The cross section for the recombination with $\mathrm{Ar}_{2}^{+}$is given by Bretagne [24], while the cross section for the recombination with $\mathrm{Ar}_{3}^{+}$needed to be calculated from the rate coefficient given in [29]. Assuming the cross section also has the form

$$
\sigma_{r e c, A r_{3}^{+}}(E)=\frac{\sigma_{0}}{E^{\alpha}},
$$

$\sigma_{0}=2.4 \times 10^{15} \mathrm{~cm}^{2}$ and $\alpha=1.23$ were found to reproduce the known rate coefficient best. This form for the cross sections leads to an infinite cross section at zero energy. However, because of the finite minimal energy used in the discrete EEDF, this problem is avoided.

The elastic collisions with neutrals and coulomb collisions between electrons were treated using the Rockwood formalism [22] which uses the flows of electrons in energy space caused by elastic collisions with neutrals $J_{\text {neutral }}$ and by coulomb collisions $J_{\text {coul }}$ to formulate the Boltzmann equation:

$$
\left.\frac{d f(E, t)}{d t}\right|_{\text {elastic }}=-\frac{d J_{\text {neutrals }}(E, t)}{d E}-\frac{d J_{\text {coul }}(E, t)}{d E} .
$$

When applied to a discrete EEDF, this yields for the k-th energy interval

$$
\frac{d f_{k}}{d t}=\left(a_{k-1}^{\text {neutral }}+a_{k-1}^{\text {coul }}\right) f_{k-1}+\left(b_{k+1}^{\text {neutrals }}+b_{k+1}^{\text {coul }}\right) f_{k+1}-\left(a_{k}^{\text {neutral }}+a_{k}^{\text {coul }}+b_{k}^{\text {neutral }}+b_{k}^{\text {coul }}\right) f_{k},
$$

where $a_{k}$ describes the flow of electrons to the next highest energy interval and $b_{k}$ to the next lowest. To numerically calculate these coefficients, the method by Elliott and Greene [25] was used. 


\subsection{Treatment of the rate equations}

When formulating the rate equations for the various Argon species, one has to use rate coefficients instead of cross sections. For processes without the involvement of electrons, those can be directly found in tables 2-4. If electrons are participating in the process, the rate coefficients are calculated by a convolution of the cross sections and the EEDF:

$$
k_{\text {process }}=\int \mathrm{v}(E) \sigma_{\text {process }}(E) f(E) d E
$$

The rate equations then become:

$$
\begin{aligned}
& \frac{d n_{A r^{+}}}{d t}=k_{i o n, g s} n_{g}+\sum_{l} k_{i o n, l} n_{l}+\sum_{k, l} k_{P e n n} n_{l} n_{k}-k_{1} n_{g}^{2} n_{A r^{+}} \\
& \frac{d n_{A r_{2}^{+}}}{d t}=k_{1} n_{g}^{2} n_{A r^{+}}+k_{3} n_{g} n_{A r_{3}^{+}}+k_{i o n, e x c} n_{A r_{2}^{*}}-k_{r e c, A r_{2}^{+}} n_{A r_{2}^{+}}-k_{2} n_{g}^{2} n_{A r_{2}^{+}}, \\
& \frac{d n_{A r_{3}^{+}}}{d t}=k_{2} n_{g}^{2} n_{A r_{2}^{+}}-k_{r e c, A r_{3}^{+}} n_{A r_{3}^{+}}-k_{3} n_{g} n_{A r_{3}^{+}}, \\
& \frac{d n_{A r_{2}^{*}}}{d t}=k_{4} n_{g}^{2} \sum_{l, 1 s} n_{l}-k_{\text {ion,exc }} n_{A r_{2}^{*}}-k_{\text {sel,exc }} n_{A r_{2}^{*}}-k_{5} n_{A r_{2}^{*}}, \\
& \frac{d n_{l}}{d t}=k_{\text {exc }, g s} n_{g}+\sum_{k<l} k_{\text {exc }, k l} n_{k}+\sum_{k>l} k_{\text {sel }, k l} n_{k}+\sum_{k>l} A_{k l} n_{k}+n_{g} \sum_{k>l} k_{\text {quench, } k} n_{k}+n_{g} \sum_{k} k_{k \rightarrow l}^{\text {Pop.transf }} n_{k} \\
& +\frac{k_{r e c, A r_{2}^{+}} n_{A r_{2}^{+}}}{14}+\frac{k_{r e c, A r_{3}^{+}} n_{A r_{3}^{+}}}{14}-k_{s e l, g s} n_{l}-\sum_{k>l} k_{e x c, l k} n_{l}-\sum_{k<l} k_{s e l, l k} n_{l}-k_{i o n, l} n_{l}-\sum_{l>k} A_{l k} n_{l} \\
& -\sum_{k} k_{\text {Penn }} n_{k} n_{l}-n_{g} k_{\text {quench, },} n_{l}-n_{g} \sum_{k} k_{l \rightarrow k}^{\text {Pop.transf }} n_{l} \text {. }
\end{aligned}
$$

The factor $1 / 14$ in the terms describing the recombination is due to the assumption, that one of the argon atoms created in the recombination process is in an excited state afterwards, and that the possibility for being in a specific state is equal for each of the 14 individual states. This is a rather simplified model for the dissociative recombination, since in general also two ground state atoms can be produced and the excited states need not to be populated equally. However, since the branching 
ratios are not well known, we use this simplification. Anyway, the exclusion of the production of ground state atoms has little effect, because the contribution of recombination to the population distribution is small compared to the contribution by other processes like electron impact excitation and deexcitation. The rate equations are solved simultaneously using the implemented Matlab-solver for differential equation systems of the first order. For this purpose, the Boltzmann-equation for the electrons is split up into effective rate equations for the discrete energy intervals which are effectively treated as individual species.

\section{Experimental setup}

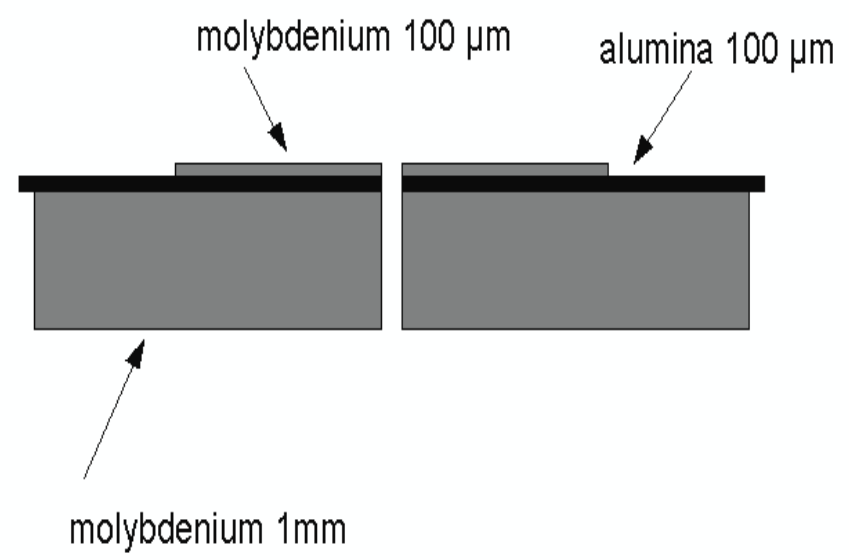

Figure 1: Schematic view of the thin cathode discharge.

Our setup differs from the usual MHCD in having asymmetric electrodes (figure 1). One of the electrodes has a larger area and is $1 \mathrm{~mm}$ thick. This electrode is usually used as anode whose shape is not essential for the operation of the discharge. The important features are the dimensions of the cathode, the dielectric layer and the diameter of the hole. In our case, the cathode and the dielectric layer are each $100 \mu \mathrm{m}$ thick, the hole has a diameter of $200 \mu \mathrm{m}$. Since high gas temperatures up to over $1000 \mathrm{~K}$ were expected [9], heat resistant materials, alumina for the dielectric layer and molybdenum for the electrodes, were used. To provide a pure argon environment, the discharge was placed inside a vacuum chamber. For optical emission spectroscopy, a quartz window and a light fibre, which guides the emitted light to a $2 \mathrm{~m}$-spectrograph, were installed on the cathode side of the vacuum chamber. The TCD is charged via the $500 k \Omega$-resistor $R_{1}$. The voltage drop over the TCD is directly measured using the high voltage probe $V_{1}$, to measure the current, a $100 \Omega$-resistor $R_{2}$ with the attached high voltage probe $V_{2}$ was added (figure 2). 


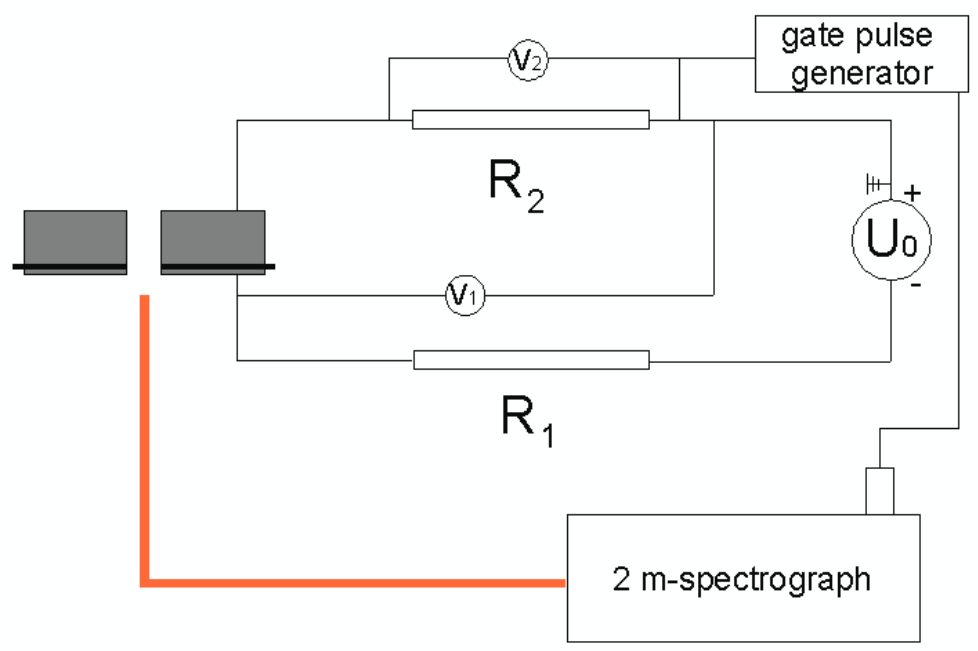

Figure 2: Schematic view of the experimental setup.

The temporally resolved measurements in the afterglow were realised using the current pulses, which occur in self-pulsing mode, to trigger the detector. For the temporally resolved measurements, the parameters were chosen so that the self-pulsing frequency is similar to the repetition rate of the detector of $20 \mathrm{kHz}$. It was found that a pressure of $900 \mathrm{hPa}$ and a supply voltage of $700 \mathrm{~V}$ fulfil this condition best, so all presented optical measurements were made with these parameters.

To measure the electron density, $1 \%$ hydrogen was added to the discharge. This way, the stark broadening of the H-Beta line at $486.13 \mathrm{~nm}$ can be used to determine the electron density using the formula

$$
\frac{\Delta \lambda}{n m}=1.03 \times 10^{-15}\left(\frac{n_{e}}{m^{-3}}\right)^{0.681}
$$

provided by Gigosos et al [21]. One has to take into account that other effects also have an influence on the measured line profiles. Usually, the line profile is a Voigt profile, which is a convolution of a Lorentzian and Gaussian profile [32]. Due to the high electron densities, pressure broadening, which yields a Lorentzian profile with a FWHM of $0.014 \mathrm{~nm}$, is negligible to the also Lorentzian-like Stark broadening [32] with a FWHM of about $1 \mathrm{~nm}$. For the Gaussian part of the profile, only the instrumental broadening of $0.118 \mathrm{~nm}$, which is much larger than the Doppler broadening of $\sim 0.01 \mathrm{~nm}$ [32], has to be taken into account. 


\section{Experimental results}

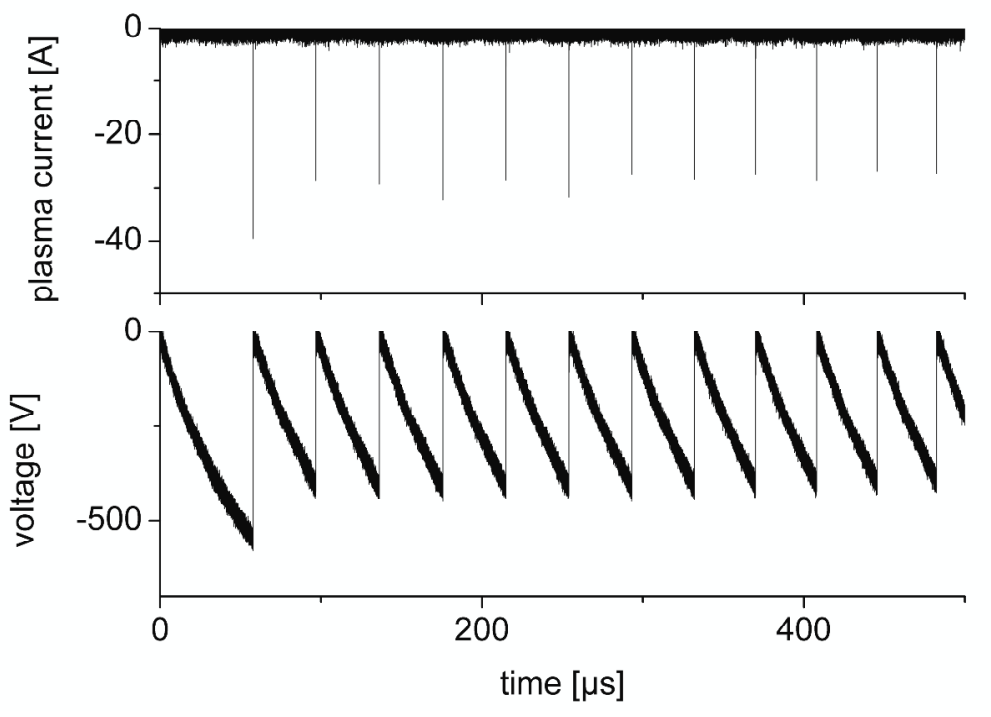

Figure 3: Self-pulsing at a pressure of $900 \mathrm{hPa}$ and a supply voltage of $1200 \mathrm{~V}$.

When operated at high pressures of several $100 \mathrm{hPa}$ and supply voltages of several $100 \mathrm{~V}$, the TCD shows a self-pulsing behaviour (figure 3) with a self-pulsing frequency of a few $10 \mathrm{kHz}$. The pulses are characterised by a sudden voltage drop and current pulses which last a few ns (figure 4). The high currents, which are reached at low voltages, indicate the ignition of a spark discharge. This is possible, because the thin cathode heats very quickly which leads to local thermionic emission. So for this selfpulsing mode, the thinness of the cathode is more important than its hollow geometry, hence the name thin cathode discharge instead of microhollow cathode discharge.

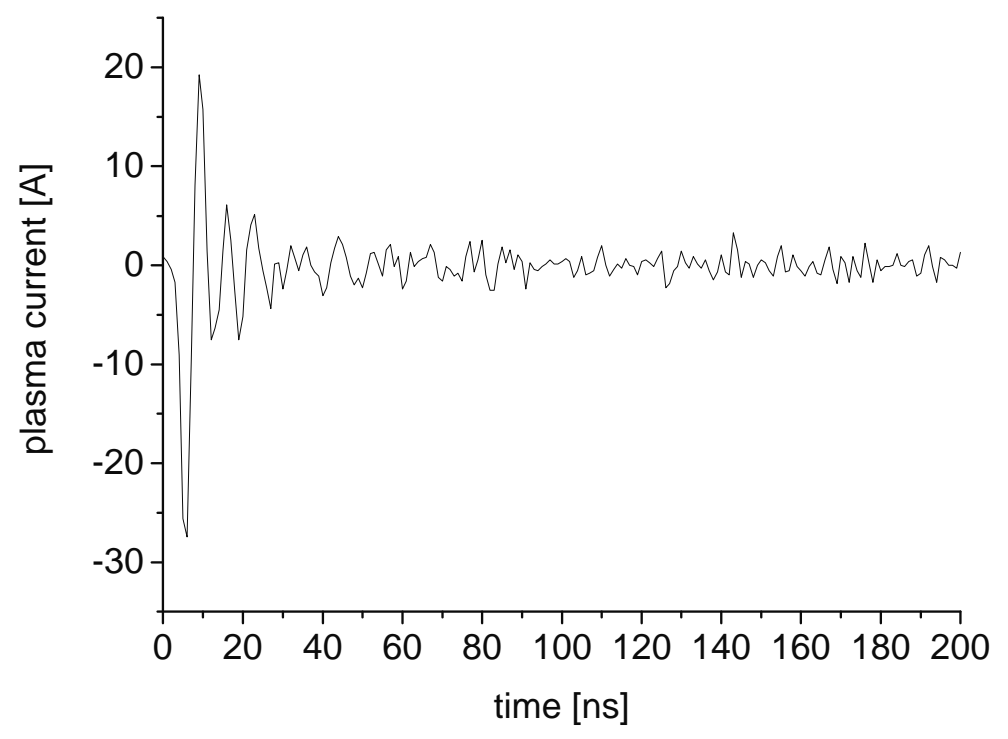

Figure 4: Zoom into one current pulse which lasts only a few ns. 


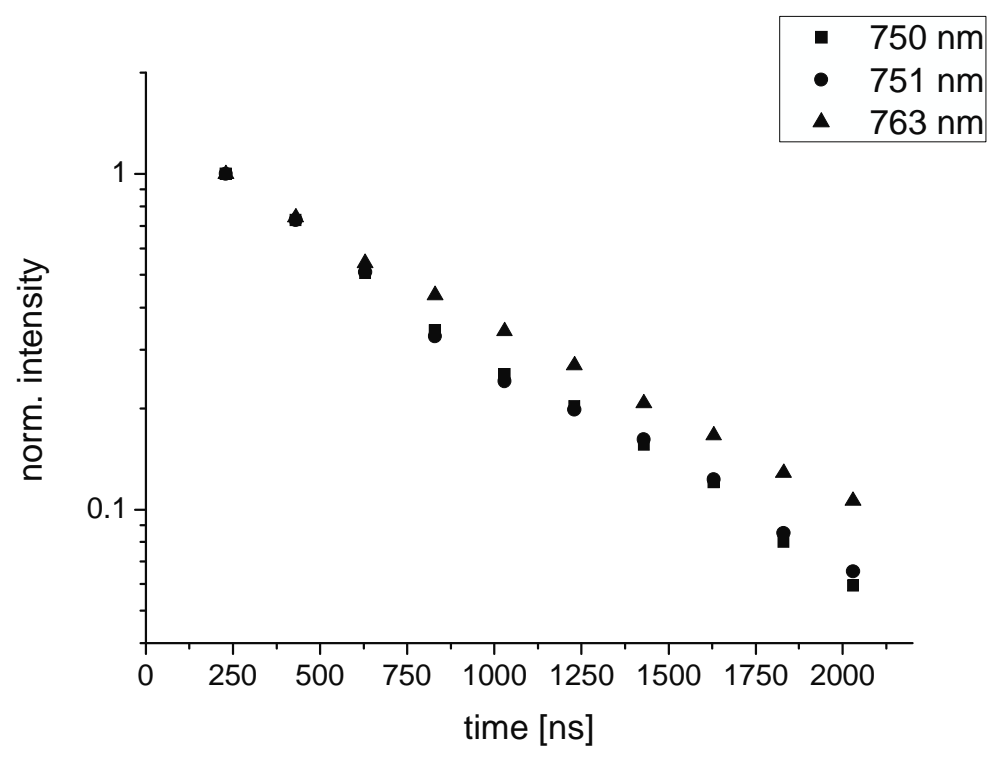

Figure 5: Normalised intensities of several 2p-lines

Figure 5 shows the temporally resolved normalised emission intensities of several lines from $2 p \rightarrow 1 s$ transitions. They all decay exponentially with lifetimes of several $100 \mathrm{~ns}$. This is well above the length of the current pulses and the natural lifetime of the $2 p$ levels, so there must still be excitation processes in the afterglow. But due to the high pressure, the electrons should cool down in a few $10 \mathrm{~ns}$ which means the electrons are heated during the afterglow. In a mixed argon-hydrogen discharge, the emission intensity also decays exponentially as well as the electron density but the decay is much faster (figure 6). This behaviour hints at the importance of argon 1s-levels for the electron heating mechanisms as they are very efficiently quenched by hydrogen [33]. Furthermore, the measured ratios of lines originating from $3 p$-states to lines originating from $2 p$-states suggest, that the EEDF is nonMaxwellian and has a high concentration of high energy electrons.
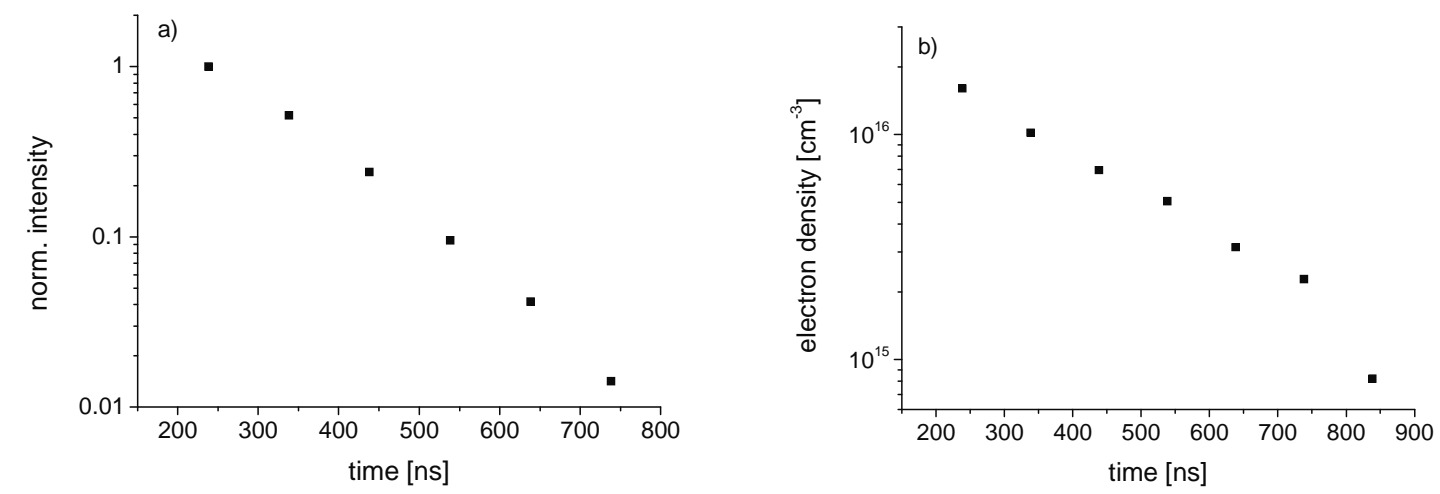

. Figure 6: a) Normalised intensity of the $750 \mathrm{~nm}$-line and b) electron density in a mixed argon-hydrogen discharge. 


\section{Simulation results and discussion}

\subsection{The early afterglow}

Before presenting the results, the initial conditions need to be discussed. Because the EEDF as well as the population densities of the excited states and molecular ions and excimers were not measured and the initial electron density could not be measured due to the internal delay of the gate pulse generator, the initial conditions are unknown. In case of the electron density, the electron density obtained by time-averaged measurements can be considered to be very close to the initial electron density as the majority of the emitted light stems from the first few ns and thus the shape of the time-averaged $\mathrm{H}$ Beta line is mainly determined by the initial electron density. The initial population densities of the excited states can be set to zero, as they are rapidly populated in a few ns due to the high ground state density and the energy stored in them is small compared to the energy provided by the electrons. In this simulation, we only considered the $1 \mathrm{~s}$ - and $2 \mathrm{p}$-states, because line ratio measurements showed, that states with higher energies are negligibly populated. The densities of the molecular ions and excimers can also be set to zero, since their formation is much slower than the ignition of the discharge, while the initial atomic ion density is equal to the electron density due to the quasineutrality of the plasma.

This leaves the unknown initial EEDF. Various tests with different EEDFs showed that the initial EEDF has a slight effect on the absolute values of the excited states, more high energy electrons lead to higher population densities, but the temporal development of the excited states do not differ in the interval in which we are interested in $(200 \mathrm{~ns}-1 \mu \mathrm{s})$, i.e. the normalised densities are independent from the initial EEDF. This can be explained by the high frequencies of the processes involving electrons, so the EEDF quickly relaxes to a characteristic form. Thus, for our purposes the initial EEDF can basically be arbitrarily chosen, as long as the mean electron energy is of the order of a few $\mathrm{eV}$. The presented results were calculated using a Maxwellian EEDF with an electron temperature of 5 $\mathrm{eV}$ and an electron density of $2.4 \times 10^{16} \mathrm{~cm}^{-3}$.

To study the influence of the gas temperature $T_{g}$, the simulation was run with different gas temperatures. As one can see in figure 7, the gas temperature has a significant influence on the lifetime of the afterglow, higher gas temperatures lead to a slower decay. This is mainly due to the $T_{g}^{-1}$ dependency of the rate coefficient for the formation of molecular ions [34], since a slower formation of these leads to a decreased recombination rate. 


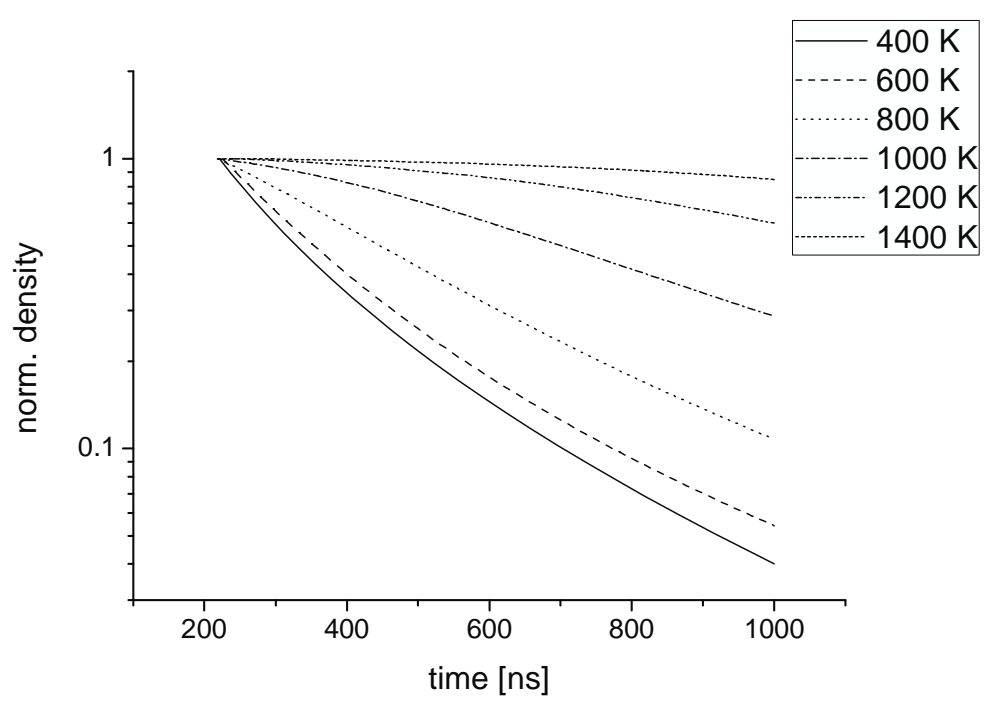

Figure 7: Normalised density of the $2 p_{1}$-level at different gas temperatures.

For the comparison of the effect of the superelastic collisions, Penning-ionization and excimer processes on the lifetime of the afterglow, the simulation was also run with only one and none these reaction types activated. The gas temperature was chosen to be $1000 \mathrm{~K}$, because with all effects activated, the decay of the $750.39 \mathrm{~nm}\left(2 p_{1} \rightarrow 1 s_{2}\right)$ line shows the best agreement with the experiment. Figure 8 shows the temporal development of the density of the $2 p_{1}$ level (the other $2 \mathrm{p}$-levels show a similar behaviour), the electron density and the mean electron energy. All processes have an influence on those parameters but contribute in different ways:

- Superelastic collisions lead to high densities of the $2 p-l e v e l s$ and a high mean electron energy, but only to slightly higher electron densities. These parameters also show a quite fast decay, in case of the $2 p-l e v e l$ density, the decay even becomes faster with time.

- Penning-ionization yields very high electron and 2 p-level densities which also decay quite slowly. The mean electron energy on the other hand is rather low, but stays nearly constant, although it starts to increase after several $100 \mathrm{~ns}$.

- Excimers have almost no influence during the first 500-600 ns. However, after this time the mean electron energy starts to rise. The 2 p-level density is rather low in the beginning, but shows a very slow decay, while the electron density is mostly unaffected.

So superelastic collisions dominate the afterglow in the beginning, but become less efficient with time due to the decaying density of the 2 p-states. Furthermore, mainly low energy electrons, which can be efficiently heated by super-elastic collisions, are lost by recombination. On the other hand, excimers 
have only little effect in the beginning, but become more important later on, when a sufficient excimer density has been built up. The loss of low energy electrons is now counter-balanced by the ongoing
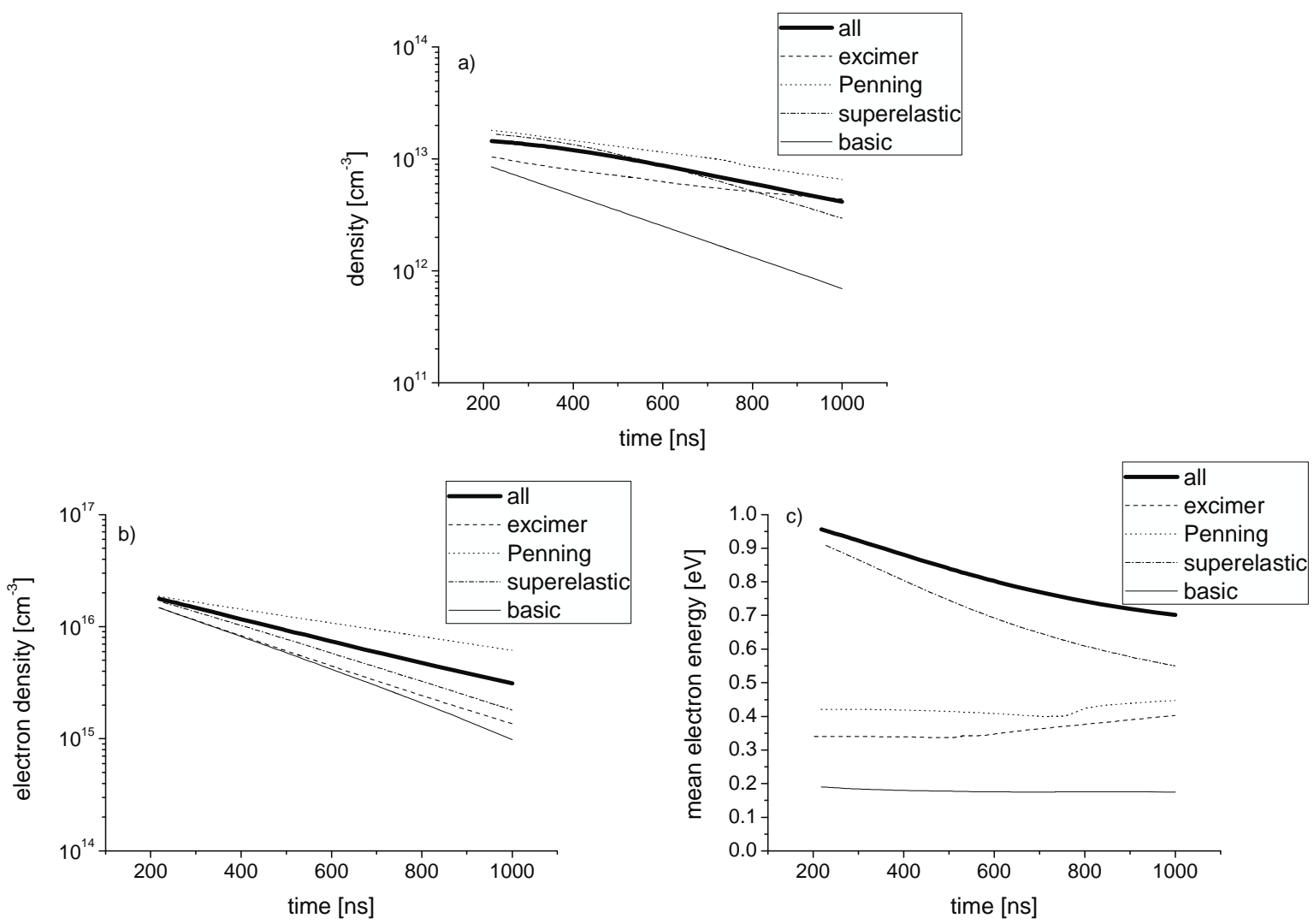

Figure 8: simulated a) population density of $2 p_{1}$-level, b) electron density and c) mean electron energy with different activated processes. The term "basic" refers to the case in which superelastic collisions, Penningionization and processes involving excimers were deactivated.

production of excimers, so the superelastic collisions with excimers stay quite efficient in contrast to superelastic collisions with excited states, which leads to lower population densities of the excited states but also to a slower decay. The Penning-ionization is a very efficient electron source, but not as efficient at heating the electrons as superelastic collisions. However, it is the most persistent mechanism as it does not rely on electrons but solely on high densities of metastables. The different roles of these mechanisms, Penning-ionization produces electrons which are heated by superelastic collisions, are confirmed by the heating and ionization rates (figure 9). Figure 9b shows that the electron impact ionization of the excited states also contributes to the production of secondary electrons, although in case of the fast decaying 2 -states, the electron impact ionization quickly loses its efficiency. Ground state and excimer ionization are negligible due to the high ionization energy and the low density, respectively. 

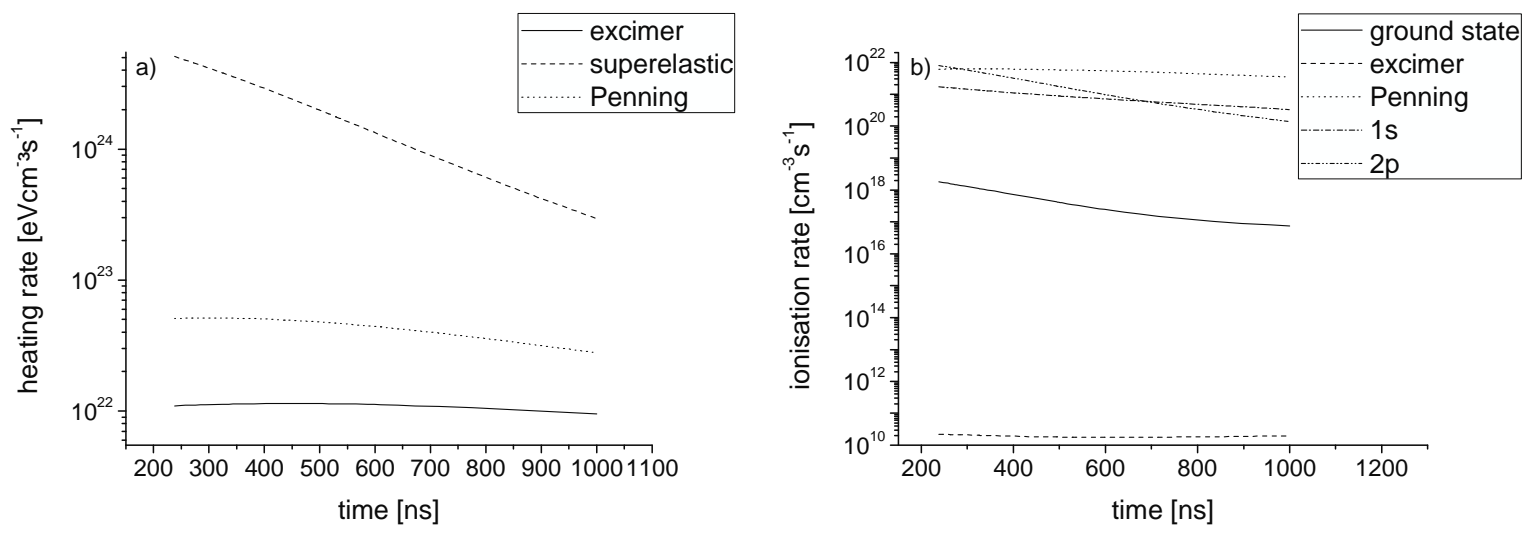

Figure 9: a) Heating and b) ionisation rates for various processes.

The inelastic nature of those processes, which prolong the afterglow, should also affect the EEDF; Penning-ionization produces electrons in very distinct energy intervals, while superelastic collisions with both excited states and excimers stepwise heat low energy electrons. On the other hand, excitation and ionization processes stepwise cool high energy electrons. Figure 10 shows the EEDFs with different effects activated at $1 \mu \mathrm{s}$. Without the heating mechanisms, the EEDF is of a Maxwellian shape, while with these effects the EEDF is only Maxwellian at low energies, since the Coulombcollisions are most effective in this regime. From energies of about $5 \mathrm{eV}$ on, the EEDFs stay nearly constant and then, after a small peak, again become Maxwellian. The energy, at which the transition from the almost constant EEDF to the Maxwellian one occurs, depends on the effect. Superelastic collisions cause a transition at about $12 \mathrm{eV}$ which is a little bit more than the energy delivered to electrons by superelastic collisions with excited atoms in a 1s-state. This pattern seems to be repeated at higher energies; at least one can see the beginning of another plateau at about $18 \mathrm{eV}$ which is again $12 \mathrm{eV}$ higher than the beginning of the first plateau. The EEDF shaped by processes involving excimers looks similar. The transition here occurs at $10 \mathrm{eV}$ which is about the energy delivered by superelastic collisions with excimers. There are additional peaks at lower energies which are likely to be caused by excitation from $1_{s}$ to $2_{p}$ - states, since the energy differences between the peaks coincide with the energy difference between these excited states. Again, this pattern is repeated at higher energies. Penning-ionization causes peaks in the energy intervals in which it creates secondary electrons, although the energy intervals, in which electrons are produced by Penning-ionization of $2 p$ levels, are less pronounced. 


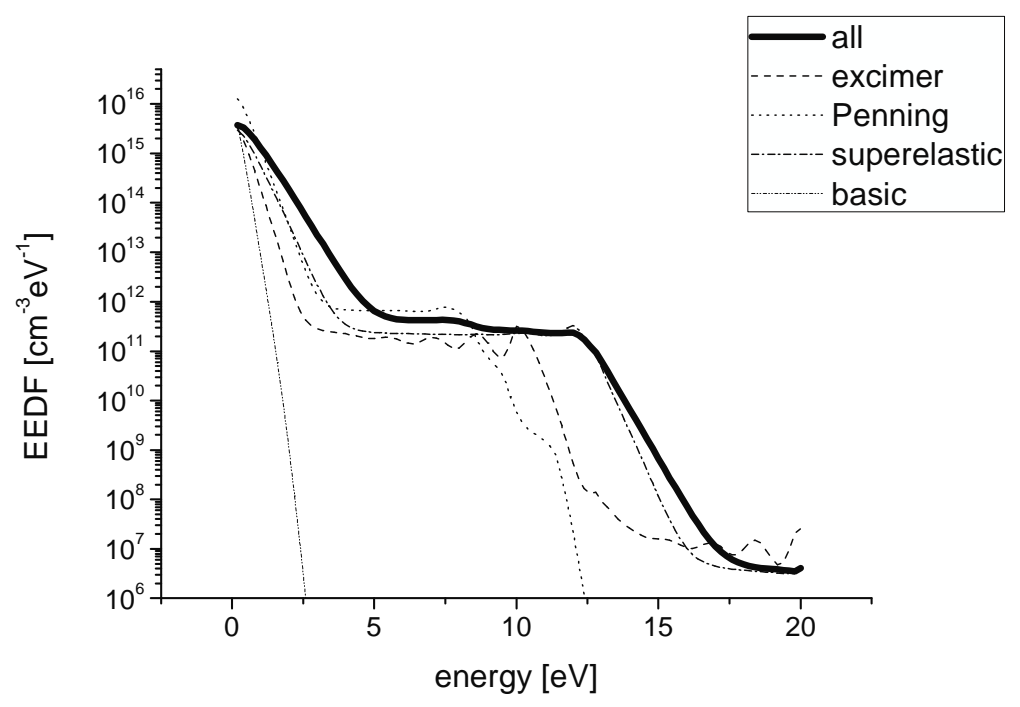

Figure 10: Simulated EEDFs at $1 \mu$ s with different activated effects. The term "basic" refers to the case in which superelastic collisions, Penning-ionization and processes involving excimers were deactivated.

These patterns are reproduced in the EEDF shaped by all processes: There are two distinct plateaus, one ends at the same energy as the plateau caused by Penning-ionization, the other ends at the same energy as the one caused by superelastic collisions. The pattern caused by excimers cannot be found which is again due to the small excimer density. These plateaus lead to a high concentration of electrons with energies above $10 \mathrm{eV}$, although the mean electron energy is quite low $(<1 \mathrm{eV})$, so the argon atoms can still be efficiently excited.

\subsection{The afterglow after $1 \mu$ s}

Because superelastic collisions with excited states become less efficient and excimers more important, it is reasonable to suspect that the afterglow develops different characteristics, once the excimer density reaches high enough values to dominate the kinetics. To verify this, the simulation was once run over the period of a whole pulse length of $50 \mu \mathrm{s}$ with all effects and a gas temperature of $1000 \mathrm{~K}$. In this calculation, diffusion was also included. 


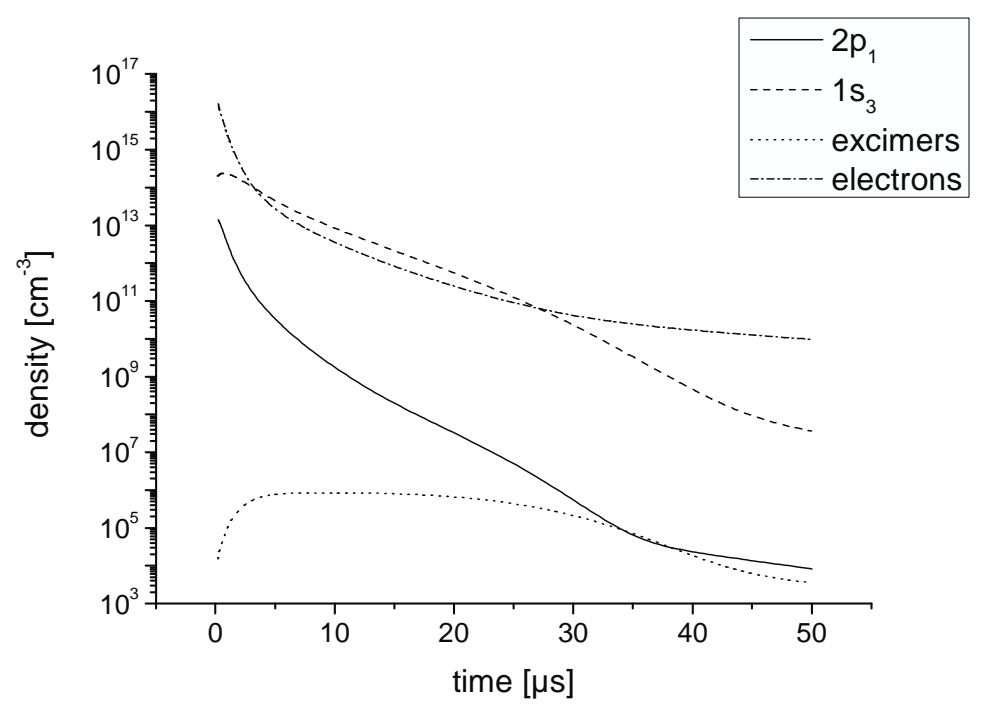

Figure 11: Densities of various species in the late afterglow.

Figure 11 shows that there is indeed a transition at about $5 \mu$ s. In the interval between about $5 \mu$ s and $30 \mu \mathrm{s}$, the electron density as well as the densities of the 2 p-states decay significantly slower than before. The excimer density stays constant and at small values, since the excimer production is basically balanced by superelastic collisions with electrons. This transition occurs when electron heating by superelastic collisions with excimers becomes more efficient than electron heating by superelastic collisions with metastables (fig. 12). Simultaneously, the mean electron energy increases again (fig. 13), since the energy, which was previously stored in metastables and excimers, is now transferred back to the electrons. This leads to increased excitation and ionization rates and thus to the slower decay of the afterglow. Furthermore, the excimers remain quite efficient while the contribution of the excited states by both superelastic collisions and Penning-ionization continues to decay fast. After about $30 \mu \mathrm{s}$, the metastable density is exhausted (see e.g. $1 s_{3}$ in fig. 12) which results in the loss of excimer production. Consequently, the excimer density decays and electron heating by superelastic collisions becomes also inefficient, leading to a steady decay of the mean electron energy. 


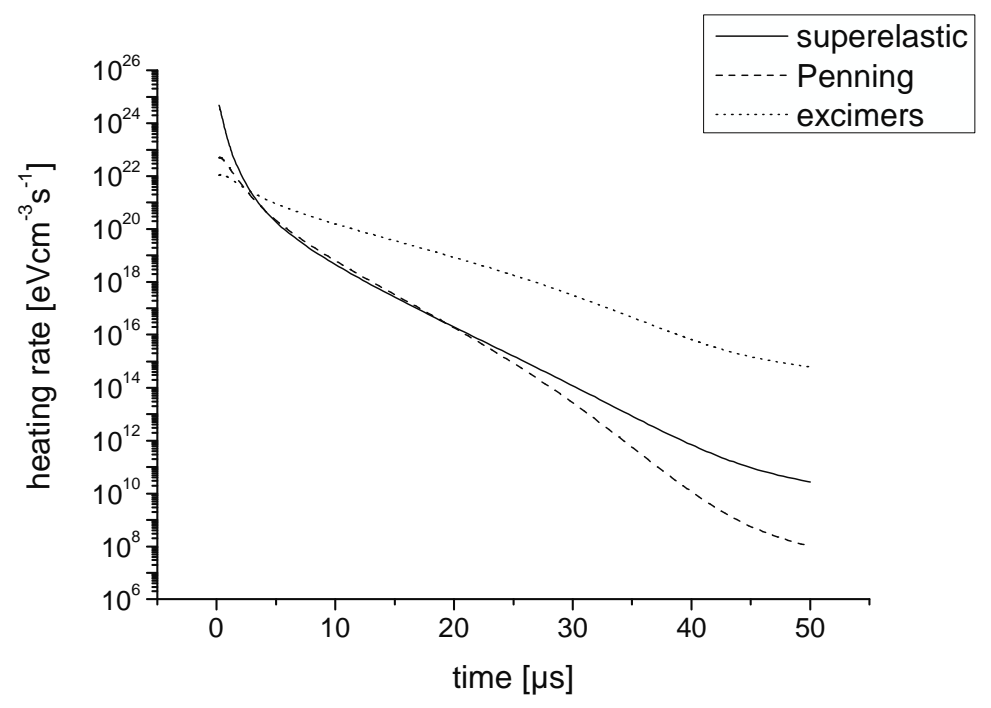

Figure 12: Heating rates in the late afterglow.

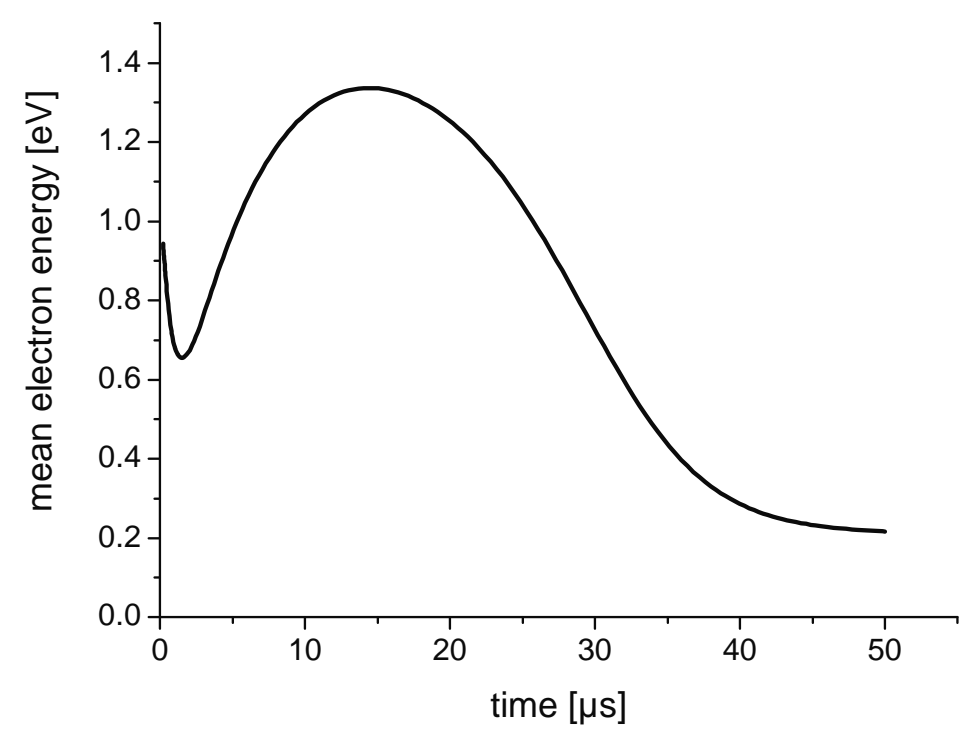

Figure 13: Mean electron energy in the late afterglow

At the end of the pulse, there are still considerable amounts of electrons, excited atoms and excimers left. This might be the reason why the breakdown voltage during self-pulsing is lower than for the first ignition. One might also suspect that these densities also affect the next afterglow but simulations done with these initial densities of the excited states and the excimers showed that this is not the case. The densities of the excited states are negligible to the densities which are created at the beginning of the pulse, while the excimers are rapidly destroyed by electron impact due to the high initial electron density. 


\section{Summary}

At high pressures of several $100 \mathrm{hPa}$, the thin cathode discharge operates in a self-pulsing mode which is caused by the repeated ignition of a spark discharge. In this mode, electron densities of several $10^{16} \mathrm{~cm}^{-3}$ are reached. The spark discharge cannot be sustained by the supplying current and thus collapses almost immediately. However, the afterglow is characterized by a long lifetime of several $100 \mathrm{~ns}$, which can only be reached if electrons are still produced and heated in the afterglow, as the electrons should cool down much faster due to the high collision frequency with the rather cold background gas.

A kinetic model of the afterglow shows, that the long living afterglow is a result of the high population density of the excited states and the formation of excimers as well as the high gas temperatures which effectively results in a lower recombination rate. The main electron source in the afterglow are the metastable 1s-levels which are subject to Penning-ionization and to a lesser extent to electron impact ionization which is much more efficient than the ground state ionization due to the low ionization energy. The secondary electrons are heated by superelastic collisions with both excited states and excimers. Excimers are less efficient in the beginning, but become more important with time until they dominate the electron kinetics after reaching a sufficient density. Because of their ongoing production, they can sustain the discharge more persistent than the decaying excited states which leads to an even slower decay of the afterglow on a timescale of $\mu$ s, once the excimers displace the excited states as main energy source.

\section{Acknowledgments}

This work was supported by the Research Department "Plasmas with Complex Interactions" of the Ruhr-University Bochum and the DFG Research Group FOR 1123 "Physics of Microplasmas". The authors also like to thank Bernd Becker, Frank Kremer and Thomas Zierow for technical support. 


\section{References}

[1] Brandenburg R, Wagner H-E, Morozov A M and Kozlov K V 2005 J. Phys. D: Appl. Phys. 381649

[2] Massoud N, Martus K and Becker K 2005 J. Phys. D: Appl. Phys. 381674

[3] Sekiguchi H, Ando M and Kojima H 2005 J. Phys. D: Appl. Phys. 381722

[4] Knake N, Reuter S, Niemi K, Schulz-von der Gathen V and Winter J 2008 J. Phys. D: Appl. Phys. 41194006

[5] Sankaran R M and Giapis K P 2002 J. Appl. Phys. 922406

[6] Urabe K, Morita T, Tachibana K and Ganguly B N 2010 J. Phys. D: Appl. Phys. 43095201

[7] Sakiyama Y and Graves D B 2009 Plasma Soures. Sci. Technol. 18025022

[8] Goree J, Liu B and Drake D 2006 J. Phys. D: Appl. Phys. 393479

[9] Penache C, Miclea M, Bräuning-Demian A, Hohn O, Schössler S, Jahnke T, Niemax K and Schmidt-

Böcking H 2002 Plasma Sources Sci. Technol 11476

[10] Lazzaroni C, Chabert P, Rousseau A and Sadeghi N 2010 J. Phys. D : Appl. Phys. 43124008

[11] Miclea M, Kunze K, Heitmann U, Florek S, Franzke J and Niemax K 2005 J. Phys. D: Appl. Phys 381709

[12] Schoenbach K H, Verhappen R, Tessnow T, Peterkin F E and Byszewski W W 1996 Appl. Phys. Lett 6813

[13] Schoenbach K H, El-Habachi A, Shi W and Ciocca M 1997 Plasma Sources Sci. Technol. 6468

[14] El-Habachi A and Schoenbach K H 1998 Appl. Phys. Lett. 7222

[15] Schoenbach K H, El-Habachi A, Moselhy M M, Shi W and Stark R H 2000 Phys. Plasmas 72186

[16] Moselhy M, Shi W, Stark R H and Schoenbach K H 2001 Appl. Phys. Lett. 791240

[17] Rousseau A and Aubert X 2006 J. Phys. D: Appl. Phys 391619

[18] Aubert X, Bauville G, Guillon J, Lacour B, Puech V and Rousseau A 2007 Plasma Sources Sci. Technol. 16 23

[19] Koutsospyros A, Yin S Y, Christodoulatos C and Becker K 2005 IEEE Trans. Plasma Sci. 3342

[20] Buntat Z, Smith I R and Razali N A M 2009 J. Phys. D: Appl. Phys 42235202

[21] Gigosos M A, Gonzales M A and Cardenoso V 2003 Spectrochim. Acta Part B 581489

[22] Rockwood S D 1973 Phys. Rev. A 82348

[23] Bretagne J, Delouya G, Godart J and Puech V 1981 J. Phys. D: Appl. Phys. 141225

[24] Bretagne J, Godart J and Puech V 1982 J. Phys. D: Apply. Phys. 152205

[25] Elliott C J and Greene A E 1976 J. Appl. Phys. 72946

[26] Peterson L R and Allen, Jr. J E 1971 J. Chem. Phys. 566068

[27] Zatsarinny O and Bartschat K 2006 J. Phys. B: At. Mol. Opt. Phys. 392145

[28] Zhu X M and Pu Y K 2010 J. Phys. D : Appl. Phys. 43015204

[29] Elson E and Rokni M 1995 J. Phys. D: Appl. Phys. 29716

[30] NIST 2006 Atomic Spectra Database http://physics.nist.gov/PhysRefData/ASD/index.html

[31] Nam S K and Economou D J 2003 J. Appl. Phys. 952272

[32] Kunze H J Introduction to plasma spectroscopy 2006, Springer

[33] Bogaerts A and Gijbels R 2000 J. Anal. At. Spectrom 15441

[34] Mehr F J and M A Biondi 1968 Phys. Rev. 176322 